\title{
Stochastic RA-Network for the Nodes Functioning Analysis in the Distributed Computer Systems
}

\author{
Zhengbing Hu \\ School of Educational Information Technology, Central China Normal University, Wuhan, China \\ E-mail: hzb@mail.ccnu.edu.cn \\ Vadym Mukhin, Heorhii Loutskii, Yaroslav Kornaga \\ National Technical University of Ukraine "Kiev Polytechnic Institute" Kiev, 03056, Ukraine \\ E-mail:v.mukhin@kpi.ua
}

\begin{abstract}
In the paper is described the simulating process for the situations analysis and the decisions making about the functioning of the Distributed Computer Systems (DCS) nodes on the basis of special stochastic RA-networks mechanism. There are presented the main problems in the estimations of the DCS nodes functioning parameters and there are shown that the suggested RA-networks mechanism allows simulate the data flow with the different, including the significantly different intensities, what is particularly important in for the situations analysis and the decisions making in the DCS nodes parameters dynamics control.
\end{abstract}

Index Terms - Distributed computer systems, parameters analysis, decisions making, RA-networks.

\section{INTRODUCTION}

One of the most important aspects in the design of the distributed computer systems (DCS) is an analysis of their functional parameters. The DCS has a special job scheduler, which, in particular, performs the distribution of the resources between the tasks, including the tasks related to DCS security ensuring [1-3]. The malfunction of job scheduler, in particular, the delays in the critical situations analysis and in the decisions making as response to them, especially in the case when there are processing the confidential information, potentially resulting in inadequate reaction or even to a complete cessation of DCS functions [1-6].

In fact, the problem of the support the sustainable functioning of the DCS is associated with an estimate of the time of the situation analysis and decision-making in DCS job scheduler [7-11]. The probability of exceeding of the maximum allowed time for the situation analysis and decision-making is functionally linked with the values of the upper and lower bounds of the allowable period for the situation analysis and decision making [10, 11]. To analyze the probability of exceeding of the critical time of decision-making, i.e., the reaction of the job scheduler to the emergency situations in the conditions of dynamic changes of the DCS settings, the effective approach, as it seems, is to perform the formalization of processes in the DCS job scheduler, in particular, on the basis of special networking mechanisms and models.

The simulation results, was received with the classical stochastic Petri networks mechanisms [12-14] for the evaluation of critical time in the situations analysis and the decision-making in the DCS job scheduler have shown that these networks allow correctly process only those cases when the events are arriving with the intensities which are practically equivalent. In the case, when the events are arriving with the different intensities, the DCS component with the smallest intensity of events flow actually is delaying the decision on the modification of the all DCS parameters. So, in order to perform the firing of the synchronized transition, which launching the process of situation analyzing and the decision making on the DCS functioning, there is required a tokens in the all input positions, due to the need to take into account the current state of the all considered DCS components to produce a complete and objective conclusion about the situation to perform the subsequent effective analysis. As a result, this synchronized transition implements the synchronization of the processes of data accumulation for the analysis, and this leads to the above mentioned problems.

However, in practical applications it is happen often enough that a violation of the DCS functions will be caused by a single component or a subset of the components. The number of events, arriving from this component is significantly exceeds the similar parameter in comparison to the other components. At the same time, the mechanisms for the sustainable supporting of the DCS functioning should quickly respond to these situations [7-11, 15-17]. Thus, there is the need to develop and use the specific networking mechanisms that will work correctly with the differential intensities of the arriving events related to the DCS components functions [18]. 


\section{THE MAIN CONCEPTS OF THE STOCHASTIC RA- NETWORKS THEORY}

To solve this problem, we suggest the mechanism of the stochastic RA-networks, which is the modification of the classic Petri-networks [12-14], and which allow handle properly the events flow with the different intensity, even with substantially different.

Let us consider the main definitions and concepts of the stochastic RA-networks theory. [18]:

Statement. RA-networks is a tuple $(S, M, L, W)$, where

- $\mathrm{S}$ - the structure of RA-networks, $N=(P, T, I, O)$, where: $P=\left\{P_{1}, P_{2}, \ldots, P_{n}\right\} \quad$ - set of places; $T=\left\{T_{1}, T_{2}, \ldots, T_{m}\right\} \quad-\quad$ a set of transitions; $I=\left\{I\left(T_{1}\right), I\left(T_{2}\right), \ldots, I\left(T_{m}\right)\right\}$ - set of input transitions, $O=\left\{O\left(T_{1}\right), O\left(T_{2}\right), \ldots, O\left(T_{m}\right)\right\}$ - set of output transitions

- $M=\left\{M_{1}, M_{2}, \ldots, M_{n}\right\}$ - marking, $M_{i}$ - number of tokens at the places of RA- networks.

- $L=\left\{\lambda_{I}(T), \lambda_{2}(T), \ldots, \lambda_{m}(T)\right\}-$ the set of intensities transitions fire;

- $W=\left\{\omega_{1}(T), \omega_{2}(T), \ldots, \omega_{m}(T)\right\}$ - set of weights of the input transitions.

As the graph, the structure of RA-networks is a bipartite-oriented graph with the places, that are the places of RA-networks and the transitions between them, and the ribs - the arc joining the places and transitions, thus there can be connect heterogeneous elements only nodes and arcs .

In the general case, there are the timed and the immediate transitions in the RA-networks. Immediate transition is fired as soon as it is enabled, and the timed one - always fires with a certain time delay.

1. Any current marking of the RA-networks is defined by the vector $M=\left(M\left(P_{1}\right), M\left(P_{2}\right), M\left(P_{3}\right), \ldots, M\left(P_{n}\right)\right)$, where the elements are the tokens presence in the corresponding places of the RA-networks.

2. Firing of the classical transition (as in the classic Petri-networks [12-14]) of the RA-networks: the transition $T_{i}$ is enabled if in the current marking on the all its inputs there are at least one token:

$$
M \geq I\left(T_{i}\right)
$$

The firing transition takes only one token from each places $P i$ by one arc and places the one token by each arc in the corresponding place $P_{j}$.

3. Firing of $N$-transition of the RA-networks: this rule corresponds to the artificial neurons functioning [18]:

$$
M \geq \sum_{k=1}^{a} \omega_{k}\left(T_{i}\right) * I\left(T_{i}\right)
$$

where $\omega_{k}\left(T_{i}\right)$ - the weight of the transition input arc $T_{i}$, where: $\sum_{k=1}^{a} \omega_{k}\left(T_{i}\right)=\omega_{t h r}\left(T_{i}\right)$, and $\omega_{t h r}\left(T_{i}\right)-$ the threshold value of the $N$-transition.

Let us consider in detail the specifics of the $\mathrm{N}$ transition [18]. In general, $N$ - transition may have $a$ inputs and $b$ outputs, all inputs are weighted, i.e. each input arc is assigned a weight $\omega_{k}$. Furthermore, $N$ transition has the threshold element, which operating as chopping comparator: if the sum of the weights of incoming arcs does not exceed the threshold value, all the outputs of the $N$-transition are set to zero, otherwise the all outputs of $N$-transition receive the one token. After the $N$ - transition is fired all its inputs are set to zero. The weights of incoming arcs and the threshold value of the $\mathrm{N}$ - transition may be changed in accordance with the current configuration and nodes parameters of the certain DCS.

The scheme of the $\mathrm{N}$-transition operation is shown in Fig. 1 [18].

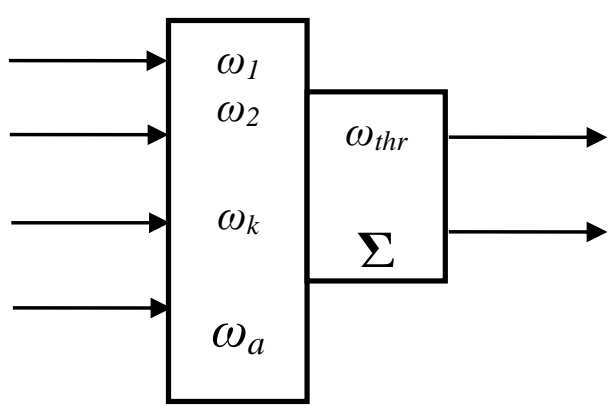

Fig.1. The Scheme of N- Transition Operation

\section{THE SPECIFICS OF THE REAL OBJECTS SiMUlation WITH THE RA-NETWORKS}

The effective simulation of the DCS inner processes is required to ensure the accuracy of the simulation and the correctness of the results analysis. The correctness of the simulation is determined by the correctness of the object model formalization, and the the analysis correctness is related to the adequacy of the properties assessment for the simulated system.

\subsection{Reachability tree and the reachability graph}

As noted before, the set of all possible markings of RA-netwoks $R\left(M_{0}\right)$ forms a reachability tree, wherein $M_{0}$ - the initial marking is a root. Starting with the root marking there are consistently defined the transitions that may be fired, the transition firing leads to a new marking, which is a new element in the reachability tree.

In general, there is a problem of the unboundeness (infinite) of the reachability tree build process. There is introduced a special terminal (final) marking to solve this problem.

There are three types of terminal markings [12-14]:

deadlocked: markings, that does not allow to fire for any transition;

duplicated: marking, which are already in the reachability tree;

propagated: marking is propagated, if $M_{j} \geq M_{i}$, where $M_{i}$ - internal marking of the reachability tree. In this case, the 
operation sequence for the transitions $\delta=\left\{T_{q}, \ldots, T_{w}\right\}$, which caused transition from $M_{j}$ to $M_{i}$ marking, can be restarted, and as a result, the transition $M_{i} \rightarrow M_{j}$ may be repeated an infinite number of times, hence the tokens in marking $M_{j}$ can multiply indefinitely.

If during the process of reachability tree building there is no any multiplying markings, the RA-networks is limited. In this case the set of achievable markings is finite and can be represented as a weighted-orinted graph with the places as the elements of $R\left(M_{0}\right)$, and for each fired transitions $T_{k} \rightarrow\left(M_{i} \rightarrow M_{j}\right)$ there is existing the $\operatorname{arc}(i, j)$ with the transition $T_{k}$.

\subsection{The simulation on the matrix approach basis}

The structure of RA-networks is described, in particular, with the input $I M$ and output $O M$ markings matrix.

We define the incidence matrix $S M$ as:

$$
S M=I M-O M
$$

Next, we introduce the vector $\delta_{j}=\left(\delta_{1}, \delta_{2}, \ldots, \delta_{j}, \ldots, \delta_{m}\right)$ with dimension $m$, and all the elements of it are equal to 0 except for the element $\delta_{j}$, equal to 1 . Then the rules of the RA-networks are described as following:

1. Classic transition $T_{j}$ (as in the classic Petri-networks [12-14]) from marking $M$ is enabled if and only if:

$$
M \geq \delta_{j} * I M
$$

and $\delta_{j} * I M i$ is the $j$ - th row of the matrix $I M$.

2. $N$ - transition $T_{j}$ from marking $M$ is enabled if and only if [18]:

$$
M \geq \sum_{k=1}^{a} \omega_{k}\left(T_{j}\right) * \delta_{j} * I M
$$

3. Firing of the classical transition $T_{j}$ from $M_{i}$ (as in the classic Petri-networks) forms the marking $M_{j}$ :

$$
M_{j}=M_{i}-\delta_{j} * I M+\delta_{j} * O M=M_{i}+\delta_{j} * S M
$$

4. Firing $N$ - transition $t_{j}$ from marking $M_{i}$ forms the marking $M_{j}[18]$ :

$$
M_{j}=M_{i}-\sum_{k=1}^{a} \omega_{k}\left(T_{j}\right) * \delta_{j} * I M+\delta_{j} * O M
$$

Thus, in the RA- networks with the initial marking $M_{0}$ and with the firing sequence of classical $\left(T_{j}, T_{l}, T_{m}\right)$ and $N$ - transition $\left(T_{n}\right)$ :

$$
T_{j} \rightarrow T_{l} \rightarrow T_{n} \rightarrow T_{m}
$$

the final marking is:

$$
\begin{gathered}
M_{f i n}=M_{i}+\delta_{j} * S M+\delta_{l} * S M- \\
-\sum_{k=1}^{a} \omega_{k}\left(T_{n}\right) * \delta_{n} * I M+\delta_{n} * O M+\delta_{m} * S M
\end{gathered}
$$

\section{MECHANISM OF THE RA-NETWORKS FOR THE SITUATIONS ANALYSIS AND DECISIONS MAKING ON THE DCS NODES FUNCTIONING}

Let us descibe the specifics of the model, which is based on the suggested RA-network, for the time assesment needed for the situations analysis and decisions making in the DCS, that includes several nodes $(N D)$.

As an example, we form the RA-network, which describe the fragment with the 5 nodes of the DCS. These components work in parallel, so the structure of the RAnetwork 5 parallel chains of events (Fig. 2).

Let us describe the tokens interpretation at the places and the events of transitions firing for this RA-network.

$P_{1}-P_{5}-$ the functioning of the nodes $N D_{1} \ldots N D_{5} ; P_{6}-$ $P_{10}$ - the data gathering for the parameters analysis of the nodes $N D_{1} \ldots N D_{5} ; P_{11}-P_{15}-$ the comparison of the data on nodes parameters analysis with the patterns of the normal functioning of the nodes $N D_{1} \ldots N D_{5} ; P_{16}$ - the module of the decisions making on the control of the nodes $N D_{1} \ldots N D_{5}$.

It should be noted that all the transitions there are timed, i.e. have a certain delay and they are interpreted as follow:

$T_{1}-T_{5}$ - the transfer of gathered data on the parameters analysis of the nodes $N D_{1} \ldots N D_{5} ; T_{6}(N-$ transition $)-$ start of the procedure of the comprehensive analysis of the gathered statistical data data on the parameters analysis of the nodes $N D_{1} \ldots N D_{5} ; T_{7}-T_{11}$ - the transfer of the results of the data analysis to module of decision making to control the functioning on the nodes $N D_{1} \ldots$ $N D_{5} ; T_{12}-T_{16}$ - the start of modification process of the nodes $N D_{1} \ldots N D_{5}$ parameters.

The operation of the DCS nodes in the RA-network is simulated as removing/adding of the tokens from/on the corresponding places $\left(P_{i}\right)$.

The structure of the RA-network has the arcs, that are opening the transitions $T_{12}-T_{16}$, so this allow to open those transitions that are connected with the DCS nodes, which are already collecting he required volume of the statistical data for the situation analysis and the decision making. This approach allows vary the decisions making on the parameters control of the various DCS nodes, i.e. to change parameters for those nodes, for which there is need in a change of the current parameters.

Transition $T_{6}$ is $N$ - transition with weights $w_{1}, w_{2}, w_{3}$ (Fig. 2). In RA- network there exists a flexible way to adjust the required volume of the analyzed data and the number of events related to the nodes. This adjustment is performing by setting of the weights $w_{i}$ coefficients of the incoming arcs and of the threshold $w_{t h r}$ for the $N$ transition firing.

Let us describe the process of the time evaluation of the situations analysis and decisions making on the basis of the RA-network mechanism. If the intensity of the 
events flow, related to the DCS nodes functioning is nearly the same, this case can be effectively processed with a classic Petri-network. But in the case, if the one (or several, but not all) of the DCS nodes has the events arrival intensity is much higher than the intensity of the events arrival by the other nodes, there is a situation when the data (tokens) will be accumulated in the place $P_{6}, P_{7}$, etc. An important condition for effective DCS nodes parameters analysis is the in-time decision making on their control, for which there is collected the certain volume of the statistical data, that, as noted before, is almost impossible in classical stochastic Petri-network, due to the fact that in these case the transition $T_{6}$ is not firing, according to the rules of transitions firing, and thus, the decision making will be delayed.

In the RA-network, this problem is solved with $\mathrm{N}$ transition, which is firing by a special condition: the transition is firing if the threshold value is reach, regardless of from which transition the data (tokens) was received. Thus, if the number of tokens in the place $P_{6}$ exceeds or equal to the threshold level (e.g., 5), and the number of tokens at the places $P_{7}-P_{10}$ is zero, then the transition $T_{6}$ is firing. However, according to the RAnetwork structure from the transitions $T_{12}-T_{16}$ the $T_{12}$

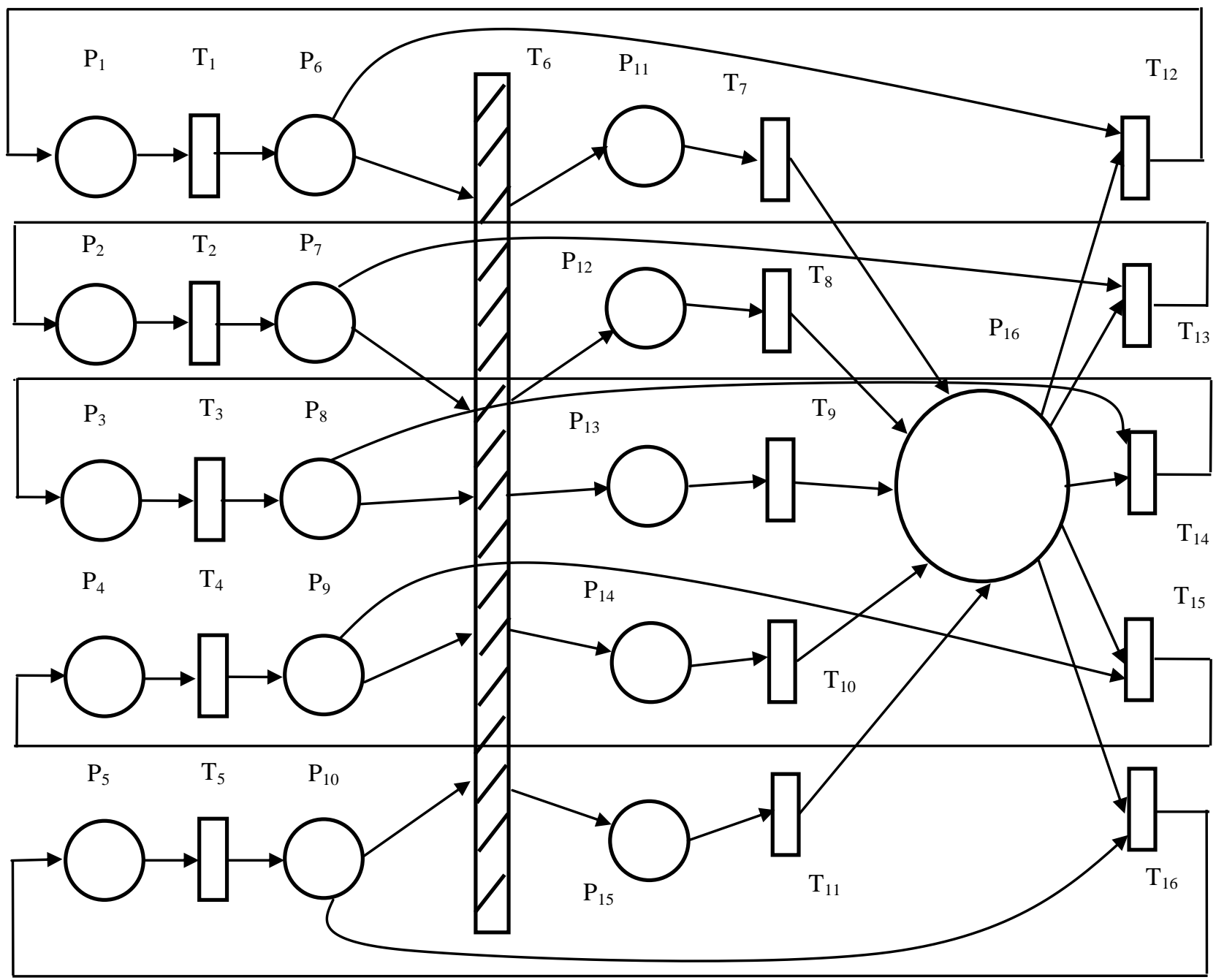

Fig.2. The Structure of RA-Network for the Time Estimation of the Situations Analysis and Decisions Making in DCS

only is firing. This means that the decision making on the nodes parameters control can be accepted the only for the DCS nodes, for which there is a sufficient volume of the statistical data. The similar case takes place if the intensity of data gathering by two chains of the RAnetwork is much higher than the intensity of data gathering by the other three chain. The decisions making on the nodes parameters control are accepted for the two relevant DCS nodes.

Let us describe the simulation results for the case when the classical Petri-network fall into the deadlock. The start marking is: $M_{0}=(1,1,1,0,0,2,2,1,0,0,0,0,0,0$, $0,0)$, i.e. the data are collecting the most intensively by the DCS nodes $N D_{1}, N D_{2}, N D_{3}$ corresponding to the place $P_{6}=2, P_{7}=2, P_{8}=1$ and the less intensively on the nodes $N D_{4}, N D_{5}: P_{9}=0, P_{10}=0$, and the process for the DCS nodes parameters modifying is not running $\left(P_{11}-\right.$ $P_{15}=0$ ). (Table 1).

For the experimental researches of the time of the situations analysis and decisions making with the RAnetwork mechanism we developed a specialized environment (Fig. 3). 
Table 1. The Experimental Results Obtained with the Petri-Network

\begin{tabular}{|c|c|c|c|c|}
\hline $\mathrm{N}$ & Time, s & Type & Transition & $\begin{array}{l}\text { Marking } \\
\mathrm{P}_{0} \mathrm{P}_{1} \ldots \mathrm{P}_{16}\end{array}$ \\
\hline 1 & 0.000000 & Start & & 1110022100000000 \\
\hline 2 & 0.000000 & Plan & $T 1$ & 1110022100000000 \\
\hline 3 & 0.000000 & Plan & $T 2$ & 1110022100000000 \\
\hline 4 & 0.000000 & Plan & $T 3$ & 1110022100000000 \\
\hline 5 & 0.018632 & Execute & $T 3$ & 1100022200000000 \\
\hline 6 & 0.270106 & Execute & $T 1$ & 0100032200000000 \\
\hline 7 & 0.804789 & Execute & $T 2$ & 0000033200000000 \\
\hline 8 & 0.804789 & End & & 0000033200000000 \\
\hline
\end{tabular}

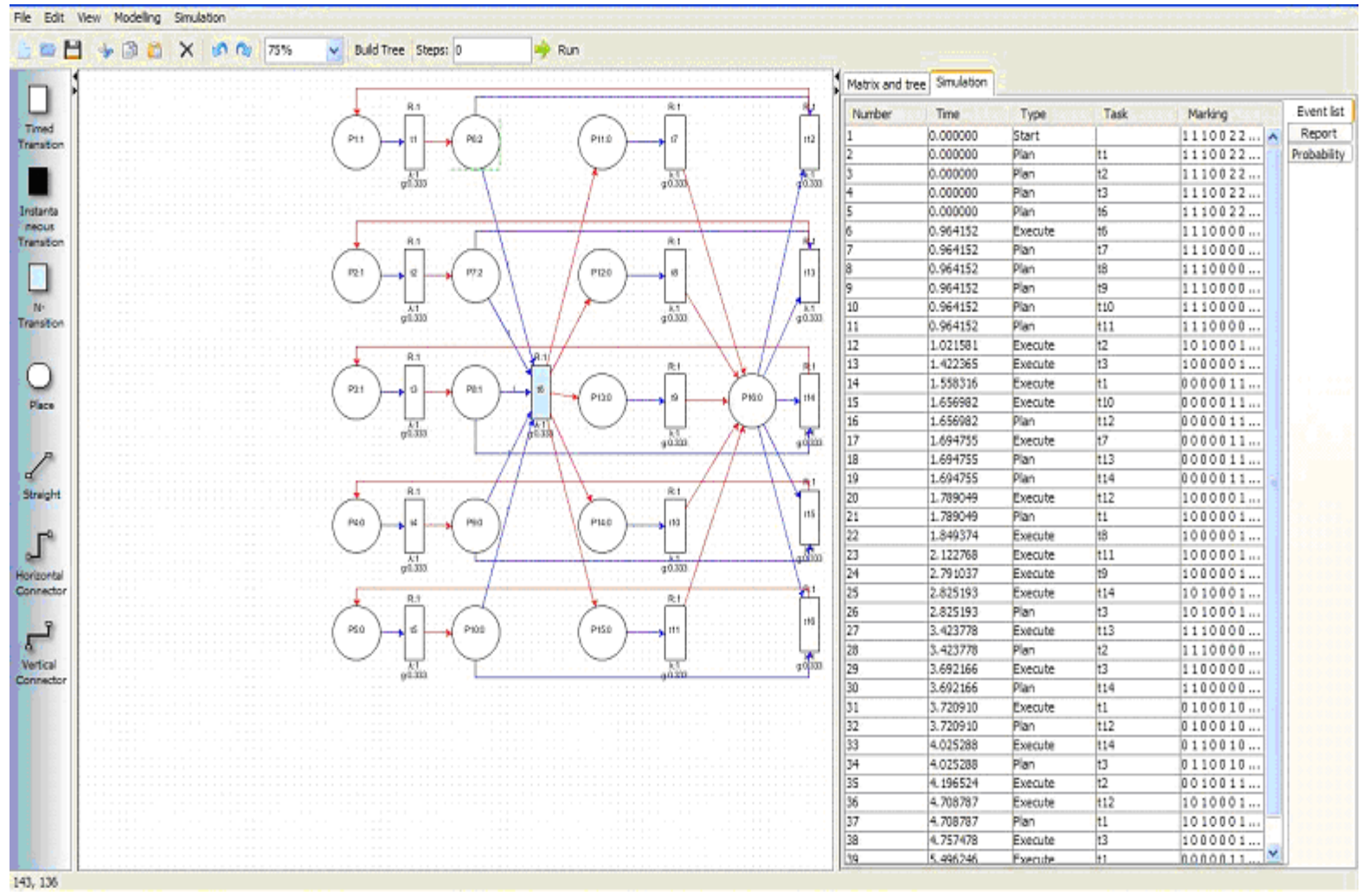

Fig.3. The Interface of the Specialized Environment with N-Transition (RA-network)

In case of the RA-network application, there are set the following weights $w_{i}$ values for the incoming arcs and threshold value $w_{t h r}$ of the $N$-transition: $w_{l}=1, w_{2}=1, w_{3}$ $=1, w_{4}=1, w_{5}=1, w_{t h r}=5$.

The simulation results are shown in the Table 2 . As it shows the Table 2, in this case, the data for the situation analysis and decisions making are accumulated in the sufficient volume for the DCS nodes corresponding to the places $P_{6}, P_{7}$ and $P_{8}$ (nodes $\left.N D_{1}, N D_{2}, N D_{3}\right)$ the places $P_{9}$ and $P_{10}$ (nodes $N D_{4}, N D_{5}$ ) has 0 tokens, i.e. this the data by this nodes are missing. In the classical Petri-network in this case the transition $T_{6}$ is not firing, and thus, the decision making on the parameters changing for the all DCS nodes is not accepted and, in fact, the simulation process finishing been fall into deadlock. 
Table 2. The Experimental Results Obtained with the RA-Network

\begin{tabular}{|c|c|c|c|c|}
\hline $\mathrm{N}$ & Time, $\mathrm{s}$ & Type & Transition & Marking \\
\hline 10 & $101 \mathrm{e}, \mathrm{s}$ & 1уре & 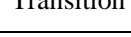 & $\mathrm{P}_{0} \mathrm{P}_{1} \ldots \mathrm{P}_{16}$ \\
\hline 1 & 0.000000 & Start & & 1110022100000000 \\
\hline 2 & 0.000000 & Plan & $T 1$ & 1110022100000000 \\
\hline 3 & 0.000000 & Plan & $T 2$ & 1110022100000000 \\
\hline 4 & 0.000000 & Plan & $T 3$ & 1110022100000000 \\
\hline 5 & 0.000000 & Plan & T6 & 1110022100000000 \\
\hline 6 & 0.964152 & Execute & T6 & 1110000000111110 \\
\hline 7 & 0.964152 & Plan & $T 7$ & 1110000000111110 \\
\hline 8 & 0.964152 & Plan & $T 8$ & 1110000000111110 \\
\hline 9 & 0.964152 & Plan & $T 9$ & 1110000000111110 \\
\hline 10 & 0.964152 & Plan & $T 10$ & 1110000000111110 \\
\hline 11 & 0.964152 & Plan & $T 11$ & 1110000000111110 \\
\hline 12 & 1.021581 & Execute & $T 2$ & 1010001000111110 \\
\hline 13 & 1.422365 & Execute & $T 3$ & 1000001100111110 \\
\hline 14 & 1.558316 & Execute & $T 1$ & 0000011100111110 \\
\hline 15 & 1.656982 & Execute & $T 10$ & 0000011100111011 \\
\hline 16 & 1.656982 & Plan & $T 12$ & 0000011100111011 \\
\hline 17 & 1.694755 & Execute & $T 7$ & 0000011100011012 \\
\hline 18 & 1.694755 & Plan & $T 13$ & 0000011100011012 \\
\hline 19 & 1.694755 & Plan & $T 14$ & 0000011100011012 \\
\hline 20 & 1.789049 & Execute & $T 12$ & 1000001100011011 \\
\hline 21 & 1.789049 & Plan & $T 1$ & 1000001100011011 \\
\hline 22 & 1.849374 & Execute & $T 8$ & 1000001100001012 \\
\hline 23 & 2.122768 & Execute & $T 11$ & 1000001100001003 \\
\hline 24 & 2.791037 & Execute & $T 9$ & 1000001100000004 \\
\hline 25 & 2.825193 & Execute & $T 14$ & 1010001000000003 \\
\hline 26 & 2.825193 & Plan & $T 3$ & 1010001000000003 \\
\hline 27 & 3.423778 & Execute & $T 13$ & 1110000000000002 \\
\hline 28 & 3.423778 & Plan & $T 2$ & 1110000000000002 \\
\hline 29 & 3.692166 & Execute & $T 3$ & 1100000100000002 \\
\hline 30 & 3.692166 & Plan & $T 14$ & 1100000100000002 \\
\hline 31 & 3.720910 & Execute & $T 1$ & 0100010100000002 \\
\hline 32 & 3.720910 & Plan & $T 12$ & 0100010100000002 \\
\hline 33 & 4.025288 & Execute & $T 14$ & 0110010000000001 \\
\hline 34 & 4.025288 & Plan & $T 3$ & 0110010000000001 \\
\hline 35 & 4.196524 & Execute & $T 2$ & 0010011000000001 \\
\hline 36 & 4.708787 & Execute & $T 12$ & 1010001000000000 \\
\hline 37 & 4.708787 & Plan & $T 1$ & 1010001000000000 \\
\hline 38 & 4.757478 & Execute & $T 3$ & 1000001100000000 \\
\hline 39 & 5.496246 & Execute & $T 1$ & 0000011100000000 \\
\hline 40 & 5.496246 & End & & 0000011100000000 \\
\hline
\end{tabular}

The simulation results with the suggested RA- network (see Table 2) shows that in this case for the situation analysis and decision making there are sufficient the statistical data gathered for the 3 DCS nodes only due to the functional characteristics of $\mathrm{N}$-transition. Table 2 shows the transition $T_{6}$ was fired the several times, therefore, there was made the decisions on the parameters modifications of DCS nodes, for which the data are accumulated and the accepted decisions are transferred to the corresponding DCS nodes through the transitions $T_{12}$
- $T_{14}$. The transition $T_{15}$ and $T_{15}$ was not firing even once, since for the corresponding DCS nodes there not gathering the required statistical data, and the decision about the parameters changes for these nodes is not accepted. As a result, after the first cycle of the statistical data accumulation process will continue for the all DCS nodes and simulation of the nodes functioning continues also, that is reflected by multiple firings of the transitions $T_{12}, T_{13}$ and $T_{14}$. 


\section{CONCLUSIONS}

In the paper is presented the formal procedure for the constructing of the structure of the model based on RAnetworks simulating mechanism for the situation analysis and decision-making in the job scheduler of the DCS. The results of experimental researches shown, that the simulation of the DCS components functions, in particular, in the tasks of the security analysis on the basis of the classical Petri networks has some shortcomings and limitations. In particular, if the arriving events for the situations analysis and decision-making on the minimization of the security risks for the DCS components are distributed unevenly, the Petri-networks fall in the deadlock and, in fact, may not correctly react to the processes in this case.

There is developed the theory of the stochastic RAnetworks, where is introduced a special $\mathrm{N}$-transition, and the rule for firing it complies with the rule of functioning of artificial neuron. The proposed RA-networks can be used, in particular, for the simulation and risks analysis of the security violations in distributed computer systems.

There is presented the main theoretical background for the RA-networks in tasks of the simulation of real-world objects, as well as the properties of RA-networks and the specifics of the simulation of the real-world objects based on RA-networks are studied.

The developed specialized environment allows simulate the processes of the situation analysis and decision making in the job scheduler of the DCS, basing on the RA-networks. There is performed the simulation and experimental analysis of the parameters of the process of the situations analysis and decision-making in the DCS job scheduler using the suggested stochasticRA-networks.

It is shown that the RA-networks allow correctly perform simulation of the events flow with the various, including substantially different intensities, that is particularly important in the analysis of situations and decision-making in the dynamic control of the DCS parameters. We also shown that the proposed mechanism based on the simulation with the stochastic RA-networks allow to reveal and identify the critical components of DCS, the result of functioning of which may lead to the exceed of the allowable time for the situation analysis and decision making, what is very important for the situations analysis and decision-making processes in modern dynamic distributed computer systems.

\section{ACKNOWLEDGMENT}

The authors would like to thank anonymous reviewers for their careful reading of this paper and for their helpful comments.

This scientific work was supported by RAMECS and CCNU16A02015.

\section{REFERENCES}

[1] Hentea M. Enhancing information security risk management with a fuzzy model. Proceedings of 19th
International Conference on Computer Application in Industry and Engineering. Las Vegas, USA, 2006. PP. 132-139.

[2] Tassabehji R. Information security threats. Encyclopedia of multimedia technology and networking. IDEA Group ReferencePennsylvania: "Hershey", 2005. pp. 404-410.

[3] Wang F.Y. Agent-based control for networked traffic management systems. IEEE Intelligent Systems, № 5(19), 2005. PP. 92-96.

[4] Shan H., Smith W., Oliker L., Biswas R. Job scheduling in a heterogeneous GRID environment. http://www.osti.gov/bridge/servlets/purl/860301UfJBKm/860301.pdf

[5] Der Braber F., Hoggavink I., Lund M., Stolen K. and Vraalsen V. Model-based security analysis in seven steps. A guided tour to the CORAS method. BT Technology, vol. 1, N 25, 2007. PP. $101-117$.

[6] Dahl O.M., Wolthusen S.D. Modeling and Execution of Complex Attack Scenarios using Interval Timed Colored Petri Nets. Proc. of 4th IEEE Intern. Inform. Workshop, Royal Holloway, UK, 2006. PP.157-168.

[7] Bonald T, Feuillet M. Network Performance Analysis. 2011. 253 p. DOI: $10.1002 / 9781118602911$.

[8] Newman M.E.J. The structure and function of complex networks. SIAM Review № 45, 2003. PP.167-256.

[9] Motter A., Matias M., Kurths J., Ott E. Dynamics on Complex Networks and Applications. Physical, 2006. 224 p.

[10] Wu Y., Min G., Li K., Javadi B. Performance Analysis of Communication Networks in Multi-Cluster Systems under Bursty Traffic with Communication Locality. Proc. of Global Telecommunications Conference, 2009. PP.1-6. DOI: 10.1109/GLOCOM.2009.5425416.

[11] Dong F., Li M., Gong X., Li H., Gao F. Diversity Performance Analysis on Multiple HAP Networks. 2015. PP.15398-15418. DOI:10.3390/s150715398. www.mdpi.com/1424-8220/15/7/15398/pdf.

[12] Peterson J.L. Petri Net Theory and the Modeling of Systems. Prentice-Hall, 1981.

[13] Murata T. Petri nets: properties, analysis and applications. Proceedings of the IEEE, 77(4), 541-80, 1989.

[14] Reisig W., Rozenberg G. Lectures on Petri Nets 1: Basic Models. Springer-Verlag, 1998.

[15] Rasmi M., Al-Qerem A. PNFE: A proposal approach for proactive network forensics evidence analysis to resolve cyber crimes. International Journal of Computer Network and Information Security (IJCNIS), Vol. 7, No. 2, January 2015, PP.25-32. DOI: 10.5815/ijcnis.2015.02.03.

[16] Karuppanchetty C., Edmonds W., Kim S.-il, Nwanze N. Artificially Augmented Training for Anomaly-based Network Intrusion Detection Systems. International Journal of Computer Network and Information Security(IJCNIS), Vol. 7, No. 10, September 2015, PP.114. DOI: $10.5815 /$ ijcnis.2015.10.01

[17] Govindarajan M. Hybrid Intrusion Detection Using Ensemble of Classification Methods. International Journal of Computer Network and Information Security(IJCNIS), Vol. 6, No. 2, January 2014, PP.45-53, DOI: 10.5815/ijcnis.2014.02.07

[18] Mukhin V.Ye. The risk-based information security. Kiev, NTUU "KPI", "Polytechnica”, 2011.292 p. 


\section{Authors' Profiles}

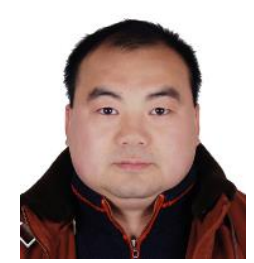

Zhengbing Hu: Ph.D., Associate Professor of School of Educational Information Technology, Central China Normal University, M.Sc. (2002), Ph.D. (2006) from the National Technical University of Ukraine "Kiev Polytechnic Institute". Postdoc (2008), Huazhong University of Science and Technology, China. Honorary Associate Researcher (2012), Hong Kong University, Hong Kong.

Major interests: Computer Science and Technology Applications, Artificial Intelligence, Network Security, Communications, Data Processing, Cloud Computing, Education Technology.

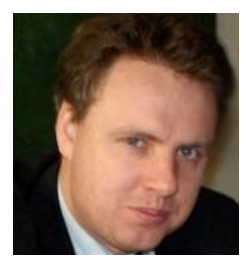

Vadym Mukhin: Professor of computer systems department of National Technical University of Ukraine "Kiev Polytechnic Institute", Doct. of Sc.

Born on November 1, 1971. M. Sc. (1994), PhD (1997), Doct. of Sc. (2015) from the National Technical University of Ukraine "Kiev Polytechnic Institute"; Assoc. Prof. (2000), Professor (2015) of computer systems department.

Major interest: the security of distributed computer systems and risk analysis; design of the information security systems; mechanisms for the adaptive security control in distributed computing systems; the security policy development for the computer systems and networks.

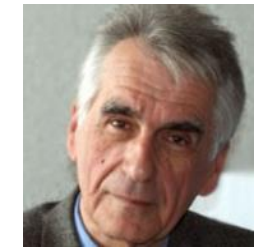

Heorhii Loutskii: Chair of computer systems department of National Technical University of Ukraine "Kiev Polytechnic Institute", Doct. of Sc.

Born on June 22, 1938. M. Sc. (1964), $\mathrm{PhD}$ (1970), Doct. of Sc. (1986) from the National Technical University of Ukraine "Kiev Polytechnic Institute"; Assoc. Prof. (1973), Professor (1987) of computer systems department, Chair (1991) of computer systems department.

Major interest: the theory of parallel processing, the theory of the pipelined data processing, an pipelined-cyclic data processing, multidimensional pipelining, systolic and nonautonomous processing; the theory of the design of the largescale high productive computing systems; the methods and mechanisms for the information security in the computer systems and networks.

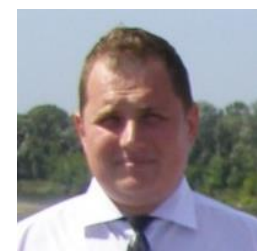

Yaroslav Kornaga: Assoc. professor of computer systems department of National Technical University of Ukraine "Kiev Polytechnic Institute", PhD.

Born on January 1, 1982. M. Sc. (2005), PhD (2015), from State University of Telecommunications; Assoc. Prof. (2015) of techical cybernetics department.

Major interest: the security of distributed database and risk analysis; design of the distributed database; mechanisms for the adaptive security control in distributed database; the security policy development for distributed database.

How to cite this paper: Zhengbing $\mathrm{Hu}$, Vadym Mukhin, Heorhii Loutskii, Yaroslav Kornaga,"Stochastic RA-Network for the Nodes Functioning Analysis in the Distributed Computer Systems", International Journal of Computer Network and Information Security(IJCNIS), Vol.8, No.6, pp.1-8, 2016.DOI: 10.5815/ijcnis.2016.06.01 